\title{
Non-Uniform Channelization Methods for Next Generation SDR PMR Base Stations
}

\author{
Álvaro Palomo Navarro, Tony Keenan, Rudi Villing, Ronan Farrell \\ Callan Institute, Electronic Engineering Department \\ National University of Ireland Maynooth (NUIM) \\ Maynooth, Ireland \\ e-mail: apalomo, tkeenan, rudi.villing, rfarrell @eeng.nuim.ie
}

\begin{abstract}
Channelization in multi-standard Software-Defined Radio base stations presents a significant challenge. In this paper, two different channelization structures designed for a multi-standard SDR base station are studied. As a basis for comparing their computational efficiency and reconfigurability, both are applied to a specific case study of a TETRA and TEDS standards base station. Uniform narrow band spectrum division followed by channel recombination demonstrates greater flexibility than a non-uniform parallel spectrum division alternative. However, computational advantages between both structures depend on the channel allocation patterns considered.
\end{abstract}

Keywords-component; SDR, non-uniform channelization, base station, TETRA, TEDS.

\section{INTRODUCTION}

At present mobile base stations handle different types of communication standards due to the evolution of wireless digital communications. This capacity is expected to increase as new generations of standards are created. Ideally they should be able to handle the transmission and reception of these heterogeneous channels whether in the same or different frequency bands. Traditionally this has been achieved using different frequency bands. However due to the technical limitations of some base stations and the limited capacity of the frequency spectrum there is a desire for standards to share the same frequency band. In this scenario dynamic non-uniform channel spectrum allocation and channelization is required to manage channels with different bandwidths and channel spacings. Classical hardware based implementations do not satisfy such requirements. Software Defined Radio (SDR) presents an alternative solution and can allow the transmission and reception of dynamic non-uniform channels through the use of appropriate non-uniform channelization techniques.

Both uniform and non-uniform channelization techniques have been presented in literature [1-4]. These uniform methods often use efficient Discrete Fourier Transform (DFT) structures, like uniform complex DFT modulated transmultiplexers. In these filter bank (FB) based structures a synthesis bank performs channel frequency multiplexing at the transmitter side and the analysis bank implements the equivalent channelization in the receiver. This represents a "symmetric" system design where affects such as amplitude and phase distortion caused in one half of the filter bank are

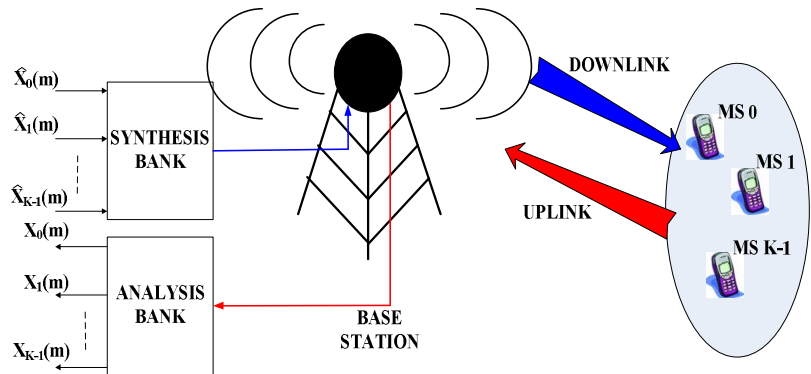

Figure 1 Asymmetric system formed by SDR base station and single carrier based mobile stations

suppressed in the complementary half. This is commonly known as perfect reconstruction. This paper examines and compares two non-uniform channelization techniques, specifically the parallel generalised DFT-FB (GDFT-FB) and the recombined GDFT-FB, and evaluates them for the specific case study of a multi-standard private mobile radio (PMR) base station (BS) implementing the Terrestrial Trunked Radio (TETRA) standard and its high-speed evolution, TETRA Enhance Data Service (TEDS) [5].

An "asymmetric" system design is depicted in Figure 1. Here the analysis and synthesis banks are applied by the base station separately on the uplink and downlink signals, whereas the mobile stations are considered to use hardware based single carrier transceivers. In this way, the system remains compliant with legacy systems. The price to be paid for this asymmetric system is that overlap between subcarriers and aliasing due to downsampling in the analysis bank must be minimized through using higher order filters. The authors are not aware of such non-uniform channelization techniques being applied to an "asymmetric" real world application with a large number of channels.

The paper is structured as follows. Section II examines the non-uniform channelization requirements of a BS, specifically focused on the TETRA/TEDS standards and possible future updates. Section III details the two nonuniform channelization methods proposed in this paper, based on the GDFT-FBs. Section IV analyses the computational load for both structures and compares them using different channel allocation patterns. Finally, Section $\mathrm{V}$ presents the results obtained for the MATLAB/Simulink implementation of both methods, and Section VI concludes the paper. 


\section{NEXT GENERATION PMR COMMUNICATIONS BASE STATIONS}

There are currently two major releases of TETRA, the first usually known as TETRA Voice \& Data (V\&D) supports $25 \mathrm{kHz}$ channels mainly allocated in the 380-400 $\mathrm{MHz}$ frequency band. The second release approved by the ETSI in 2005 is designed to support wide-band communication services using 50, 100 and $150 \mathrm{kHz}$ channels. However, the maximum throughput using TEDS is not enough to support some real time applications like video transmission. Therefore the addition of a broad-band communications system similar to the commercial $4 \mathrm{G}$ ones is under investigation [6].

In legacy BSs the antenna system can only cover the 380$400 \mathrm{MHz}$ with the addition of extra antennas constrained due to electromagnetic restrictions of the different countries [7]. As a result of this, a multiplexed frequency band solution has been widely proposed where both TETRA and TEDS channels share the $380-400 \mathrm{MHz}$ band instead of providing different bands for the two standards. Prior to the TEDS release there has been significant investment in TETRA $V \& D$ networks by different countries. Therefore it is important to ensure that any future updates are compliant with this legacy networks.

For all PMR communication standards, e.g. TETRA and TEDS, the permitted channel centre frequencies, $f_{c}(n)$ are defined by

$$
f_{c}(n)=B a n d_{-} e d g e+\left(n-\frac{1}{2}\right) \Delta f_{c}
$$

where $n$ is the channel number, Band_edge (e.g. $380 \mathrm{MHz}$ for the TETRA uplink band) is the lower edge frequency of the multiplexed frequency band, and $\Delta f_{c}$ is the channel spacing [8]. The available spectrum is divided into frequency subbands equal to the channel spacing and channels are centred within each subband.

A common hardware based implementation of the multiplexed frequency band solution would require fixed channel allocation. Due to the lack of reconfigurability of the hardware, different sections of the spectrum are allocated for specific channel sizes. This solution represents poor spectrum efficiency as no optimization of the channel allocation can be achieved. The two SDR based approaches discussed here provide a reconfigurable solution that allows for dynamic channel allocation whereby the allocation of channels of different sizes can be adapted as required. This provides the ability to use the spectrum more efficiently.

\section{NON-UNIFORM CHANNELIZATION FOR BASE STATIONS}

Channelization methods can be classified as uniform or non-uniform according to their capacity of filtering channels with equal or different bandwidths within the same frequency bands. Various non-uniform channelization techniques have been proposed in the literature, for example Frequency Response Masking non-uniform channelizers [1],

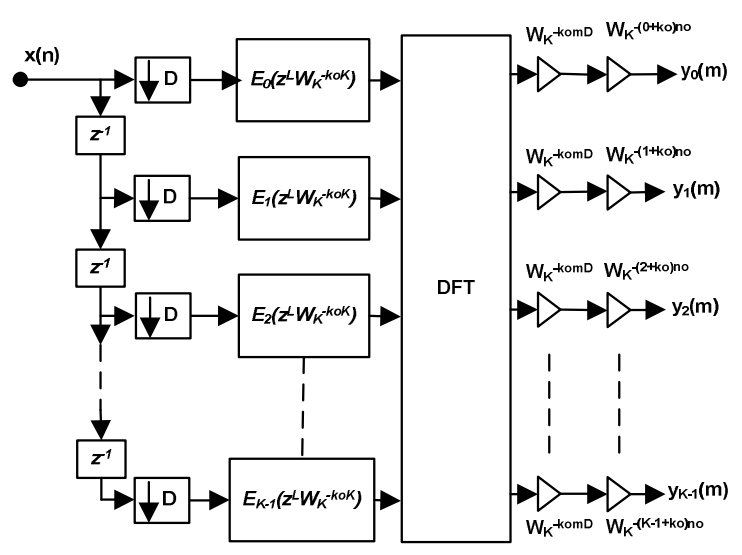

Figure 2 General DFT filter bank structure for the analysis part

and Tree Quadrature-Mirror Filter (TQMF) banks including its hybrid version [4]. However their application to real world standards, in particular studying the complexity of the structure required and range of bandwidths covered, do not appear to have been researched in detail.

In comparison, uniform channelization using polyphase DFT-FBs have been proposed for real world applications with large amount of channels due to their low complexity, e.g. multicarrier communications such as OFDM Considering this low implementation complexity, nonuniform DFT-FB based channelizers represent an attractive alternative.

Instead of the widely known DFT-FB [3], the GDFT-FB structure is used. This is due to GDFT-FB extended properties regarding the channels frequency allocation and phase response by the introduction of two parameters in the subfilters complex modulation [9]. The different $k^{\text {th }}$ filters that form the FB are obtained by complex modulation of the low-pass prototype filter $H(z)$ as

$$
H_{k}(z)=W_{K}^{-\left(k+k_{0}\right) n_{0}} H\left(z W_{K}^{\left(k+k_{0}\right)}\right)
$$

where $W_{K}=\exp (j 2 \pi / K)$ and $K$ represents the number of subchannels of the analysis bank. The parameters $k_{0}$ and $n_{0}$ determine respectively the way the different channels get stacked on the spectrum and their phase. If $k_{0}=0$ and $n_{0}=0$ the channel spectrum allocation is characterised as "even stacked" where the first channel is centred at DC. In this case the GDFT-FB structure is reduced to the classic DFT-FB. On the contrary, if $k_{0}=1 / 2 n_{0}=0$ an "odd stacked" configuration is achieved where no channels are centred at DC, but shifted a distance equal to half of the channel spacing. An odd stacked approach is used in the proposed non-uniform designs as this meets the channel allocation restrictions defined by (1). Different values of $n_{0}$ can be chosen in order to provide extra phase shifts to the FB outputs. In both even and odd stacked cases, the channel spacing is equal to

$$
\Delta f=f_{S} / K
$$


where $f_{S}$ represents the sampling frequency of the wideband multi-channel analysis bank input signal.

Considering the decomposition of the prototype filter $H(z)$ into its polyphase components according to

$$
H(z)=\sum_{i=0}^{K-1} z^{-i} E_{i}\left(z^{K}\right)
$$

where $E_{i}$ are the polyphase components [3], the rest of the subfilters $H_{k}(z)$ expressed in (2) are now obtained as

$$
H_{k}(z)=W_{K}^{-\left(k+k_{0}\right) n_{0}} \sum_{i=0}^{K-1} z^{-i} W_{K}^{-k i} W_{K}^{-k_{0} i} E_{i}\left(z^{K} \cdot W_{K}^{-k_{0} K}\right)
$$

The general implementation of the analysis GDFT-FB for both even and odd stacking cases is depicted in Figure 2. Examining (5), the different complex exponentials are applied to the different branches to perform the desired channel stacking and phase shifts. The complex exponentials $W_{K}^{-k_{0} K}$ and $W_{K}^{-k_{0} i}$ can be directly hard coded into the polyphase components of the filter bank, while $W_{K}^{-k i}$ represents the DFT algorithm. Additionally, after the DFT, a factor $W_{K}^{-k_{0} m D}$ needs to be applied in order to present the different outputs $y_{k}(m)$ centred at DC. Finally $W_{K}^{-\left(k+k_{0}\right) n_{0}}$ is applied to the outputs for phase shift purposes.

Depending on the values of the decimation factor $D$, the filter bank can be classified as critically sampled $(K=D)$ or oversampled $(K=L D)$ where $L$ represents the oversampling factor. The main benefit of an oversampled filter bank is that aliasing due to downsampling is reduced significantly. However the cost of an oversampled filter bank is the additional computational load resulting from the filter bank running at a factor of $L$ higher sample rate. This is discussed in more detail in Section IV. The two non-uniform structures to be evaluated are now described based on the above analysis.

\section{A. Parallel GDFT-FB}

The parallel GDFT-FB structure was proposed in [10] as a non-uniform channelization solution for base stations. The structure processes the wideband signal through a number of different critically decimated odd-stacked GDFT-FBs operating in parallel. Each filter bank implements a uniform division of the frequency band for a specific channel spacing and all filter banks overlap in frequency. The main focus of [10] was the application of this structure in the transmitter. In this paper, focus is now given to the implementation of this structure in the receiver.

The digitized wideband signal, with a sample rate of $f_{s}$, is fed into three parallel filter banks. Narrow band channels are extracted by selecting appropriate outputs from each of the filter banks. Any legal combination of channels can be specified by choosing the appropriate filter banks and channel numbers. Real time change in the allocation of different channels does not require redesign or re-

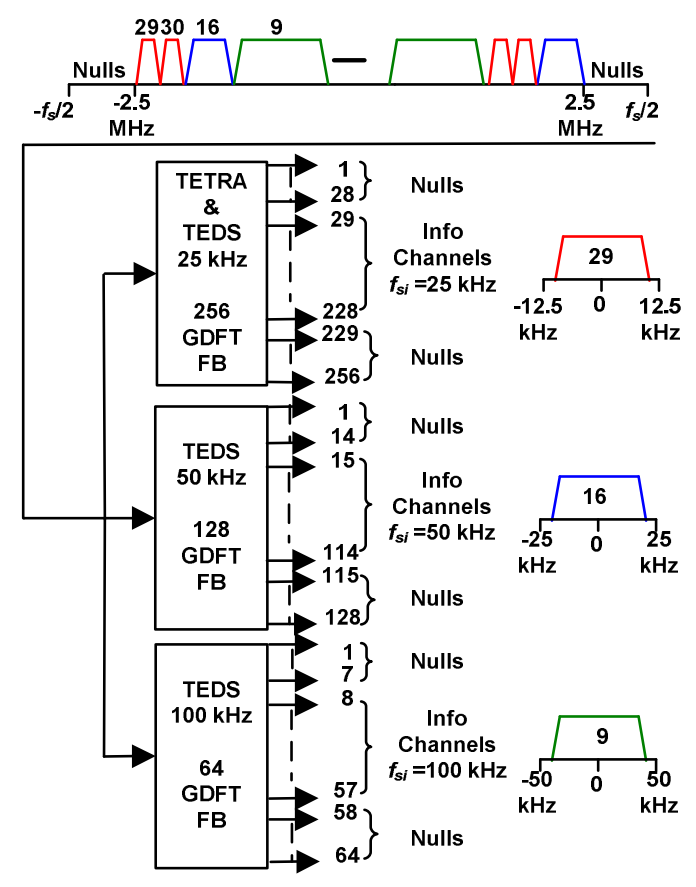

Figure 3 Parallel GDFT-FB channelization structure

optimization of the filter banks structure. Only the choice of their outputs needs to be adapted.

In Figure 3 the configuration for the $5 \mathrm{MHz}$ uplink band of the TETRA/TEDS BS case is presented. In this band, the number of channels is 200 for TETRA V\&D and TEDS 25 $\mathrm{kHz}, 100$ for TEDS $50 \mathrm{kHz}$ and 50 for TEDS $100 \mathrm{kHz}$. The DFT modulation is implemented using a power-of-2 FFT for efficiency and thus the filter bank analyses more than required bandwidth. The excess channels outside the $5 \mathrm{MHz}$ bands are set permanently as nulls.

Starting from the lower edge $(-2.5 \mathrm{MHz})$, the first two TETRA/TEDS $25 \mathrm{kHz}$ channels would be extracted from branches 29 and 30 respectively of the first transmultiplexer. The next channel shown in the multiplexed spectrum, a 50 $\mathrm{kHz}$ TEDS channel, would be selected as branch 16 of the second transmultiplexer since branch 15 refers to the same frequency range as branches 29 and 30 of the TETRA/TEDS $25 \mathrm{kHz}$ transmultiplexer. In a similar manner the next channel in the multiplexed spectrum, a TEDS $100 \mathrm{kHz}$ channel, would be selected as branch 9 of the third transmultiplexer.

Each of the GDFT-FBs in the parallel structure is critically decimated. Therefore aliasing produced by adjacent bands is increased in comparison with the oversampled case. Total suppression of the aliasing component is not necessary in this situation because the narrowband channels are not combined in a later process. However, it is necessary to reduce it to a point where it does not affect the reconstruction of the narrow band channels. In addition, since the relative phase of the different filter bank outputs is not relevant, $n_{0}$ can be made equal to zero.

This parallel configuration does not represent the best solution when the channel spectrum is not allocated 
according to (3) or when the different bandwidths of the channels are not multiples of each other. A potentially more flexible alternative non-uniform channelization method will now be considered.

\section{B. Recombined GDFT-FB}

The essential idea of a non-uniform recombination filter bank is to first analyse a signal into uniformly spaced subbands and then recombine certain groups of them to form wider bandwidths which are an integer multiple of the uniform spacing.

Non-uniform filter banks based on recombination have already been proposed in the literature related to audio and speech processing [11]. In these cases the filter banks are critically decimated, so perfect reconstruction algorithm with parameter optimization are needed in order to cancel the aliasing which results. However, due to the asymmetric configuration presented in Figure 1 this is not possible.

Oversampling ensures that aliasing in the transition bands of the channel bandpass filters is reduced in comparison with the critically sampled case. Non-uniform channelization using a recombined oversampled filter banks has been proposed in [12-13]. This recombination is carried out by the structure showed in Figure 4. Every recombined signal denoted by $y_{k, R}(m)$ is formed by $R$ contiguous subbands allocated from the $k^{\text {th }}$ output of the GDFT-FB onwards as

$$
Y_{k, R}(z)=\sum_{r=0}^{R-1} e^{j \varphi_{r}} Y_{k+r}\left(z^{M}\right) H_{M}\left(z e^{-j \beta_{r}}\right)
$$

Therefore, recombination is achieved by interpolating each of the $R$ subchannels by a factor $M$, frequency shifting by $\beta_{r}$ to the correct centre frequency and phase correction $\varphi_{r}$ in order to finally adding these shifted channels together in phase. In addition, to minimize amplitude distortion of the recombined channels, an amplitude-complementary prototype filter is required [14].

Since the GDFT-FB outputs are obtained already oversampled by a factor $L$, the interpolation factor can be chosen as $M=R / L$. Hence, the frequency and phase shifts are obtained respectively as

$$
\begin{gathered}
\beta_{r}=\pi+\frac{\pi}{R}(2 r+1) \quad \text { for } r=0, \ldots, R-1 \\
\varphi_{r}=-\left(\frac{M N}{2 D}+\frac{N_{M}}{2}\right) \beta_{r} \quad \text { for } r=0, \ldots, R-1
\end{gathered}
$$

where $N_{M}$ represents the order of the interpolation filter and $N$ is the order of the GDFT-FB prototype filter. As a possible computational improvement, the phase shift $\varphi_{r}$ operation can be placed before the interpolation. Hence it is carried out at a lower sample rate than the anti-image filtering and frequency shift operations.

As in the previous section, the filter bank is designed to cover an uplink frequency band of $5 \mathrm{MHz}$, delivering the

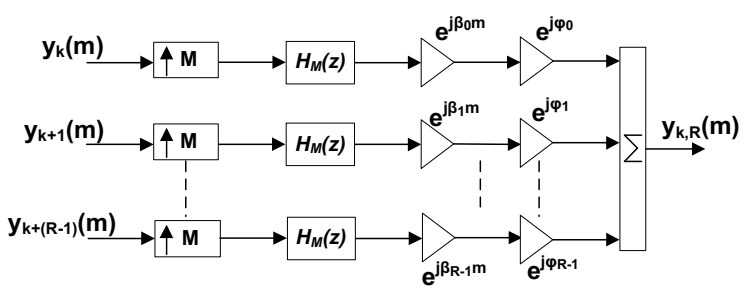

Figure 4 Recombined GDFT filter bank channelization structure

narrow band outputs with a channel spacing equal to $25 \mathrm{kHz}$. However, in this case the sample rate of the narrow band channels is twice the channel spacing, i.e. $K=2 D$, due to oversampling. TETRA/TEDS $25 \mathrm{kHz}$ channels may be selected directly from the appropriate output branches of the oversampled filter bank.

For the wider channels (50 and $100 \mathrm{kHz}$ ) adjacent output channels of the filter bank must be recombined by using the structure shown in Figure 4 . TEDS $50 \mathrm{kHz}$ channels are obtained by the recombination of two of the outputs. They do not require additional interpolation prior to frequency shifting and adding due to the original oversampling. A TEDS $100 \mathrm{kHz}$ channel is obtained by interpolating each channel by 2 and then frequency shifting and adding.

This method and the previous parallel one both have the same fundamental channelization capabilities. The parallel GDFT-FB method is best suited to schemes with relatively few possible channel bandwidths and alignment patterns (e.g. just 3 for TETRA/TEDS). The recombination method may be better suited to schemes which permit a larger variety of channel bandwidths and alignments.

\section{COMPUTATIONAL COMPLEXITY}

Apart from the requirement to dynamically filter channels with different bandwidths, computational load is the principle metric that might be used to differentiate channelization structures.

In order to compare the performance of the two structures proposed in this paper, their computational performance will be measured in terms of real multiplications and real additions per input sample for the PMR BS use case. Different configurations of TETRA/TEDS channel allocation patterns will be considered.

For the parallel GDFT-FB, the computational load remains constant independent of the number of 25, 50 and $100 \mathrm{kHz}$ channels. In this structure, all the sub-channels of all the filter banks will be processed whether they contain valid communication channels or not. The number of real multiplications per input sample for each of GDFT-FB is

$$
\frac{1}{K}\left[4(N+1)+4\left(\frac{K}{2}\left(\log _{2}(K)-1\right)\right)+4 K\right]
$$

and the number of real additions per input sample is

$$
\frac{1}{K}\left[2(2 N+1)+2\left(\frac{3 K}{2}\left(\log _{2}(K)-1\right)\right)+2 K\right]
$$


where the first term counts the arithmetic operations for the complex valued prototype filter with order $N$, the second term counts the arithmetic operations due to the complex valued $K$-point radix-2 FFT [15], and the last term counts the arithmetic operations due to multiplication by a complex exponential signal. It should be noted that the arithmetic operations due to the $K$-point FFT could be reduced through the use of more efficient FFT algorithms such as the split radix FFT algorithm. However, power-of-2 FFT algorithms such as the radix-2 and radix-4 are preferred for implementation on FPGA's in practice [16].

In comparison, the computation load of the recombined GDFT-FB is comprised of a fixed part, corresponding to the oversampled GDFT-FB structure; and a variable part whose complexity depends on the number of recombined channels. The oversampled GDFT-FB requires additional computation due to oversampling. The number of real multiplications and additions per input sample for the recombined GDFT-FB are given by

$$
\begin{gathered}
\frac{L}{K}\left[4(N+1)+4\left(\frac{K}{2}\left(\log _{2}(K)-1\right)\right)+4 K\right] \\
\frac{L}{K}\left[2(2 N+1)+2\left(\frac{3 K}{2}\left(\log _{2}(K)-1\right)\right)+2 K\right]
\end{gathered}
$$

where $L$ is the oversampling factor.

The additional number of real multiplications and real additions per input sample required for each recombination structure are given by (13) and (14) respectively, where $J$ is the number of channels recombined into a wider channel, $M$ is the interpolation factor required for recombination and $N_{i}$ is the order of the anti-alias filter required in the interpolation.

$$
\begin{gathered}
\frac{1}{K}\left[L \times M \times J\left(2 \frac{N_{i}+1}{2}+4+\frac{4}{M}\right)\right] \\
\frac{1}{K}\left[L \times M\left(J\left(2 N_{i}+2+\frac{2}{M}\right)+2(J-1)\right)\right]
\end{gathered}
$$

Considering the specific case of a TETRA/TEDS BS, a stop-band rejection of $55 \mathrm{~dB}$ is required to provide sufficient channel selectivity. Also, in order to minimize the amplitude distortion a pass-band ripple of $0.1 \mathrm{~dB}$ is selected. The length of the FIR prototype filters for each filter bank was calculated using [17]. For their design, Parks-McClellan equiripple algorithm was employed [2].

In a standard analysis-synthesis filter bank the aliasing effects can be minimized due to the relationship between the analysis and synthesis parts. However, because of the asymmetric system design only the analysis bank in the receiver is used, therefore aliasing is minimized through

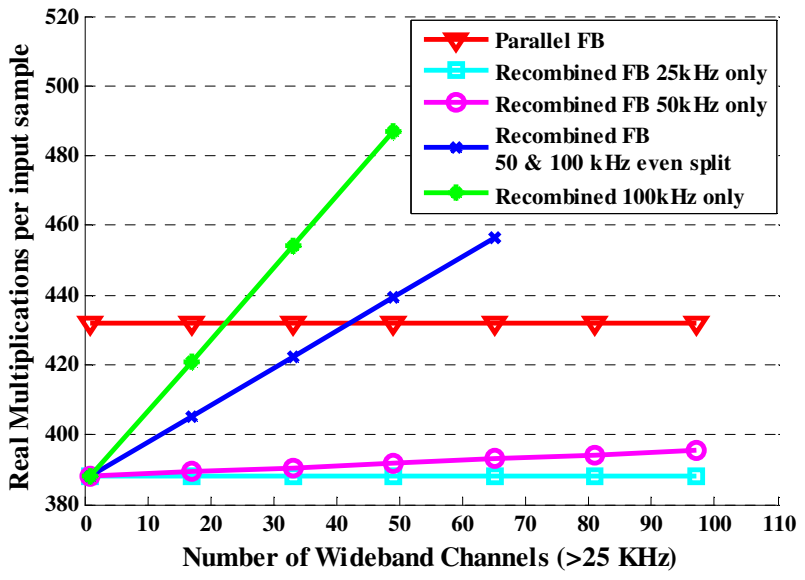

Figure 5 Computational load comparison between parallel and recombined GDFT-FB structures considering different configurations of TETRA/TEDS channel allocation patterns

tighter filter specifications. This is especially significant in the critically sampled case [18].

For both structures the filter length for the $25 \mathrm{kHz}$ filter bank prototype was calculated to be approximately 11264 taps. For the parallel DFT-FB the required prototype filter lengths for the $50 \mathrm{kHz}$ and $100 \mathrm{KHz}$ filter banks were calculated to be 3584 and 1532 respectively.

These orders represent theoretical calculations where no filter coefficient optimization has been applied. In order to decrease the length of the required filters to practical sizes, the application of other complex modulation structures such as exponential modulated filter banks (EMFB) or modified DFT-FB (MDFT-FB) with more optimized filter design methods could be studied [19].

Despite the high filter orders, by using filter banks only a single prototype filter is required compared to a per-channel hardware implementation where large independent filters are required for each narrowband channel. Also, the FB filters run at the decimated sample rate, while filters in a per channel implementation usually do it at the high sample rate of the multi-channel input signal.

Figure 4 shows the total real multiplications per sample for both structures presented for an increasing number of wideband channels, i.e. 50 and $100 \mathrm{kHz}$ channel. For the recombined GDFT-FB different splits of these wideband channels are plotted. It can be observed that the parallel GDFT-FB requires a constant number of real multiplications irrespective of the number of wideband channels. This is significantly more than the most efficient recombined GDFT-FB case where only narrowband signals are present. As the number of wideband channels in the recombined GDFT-FB increases it is obvious that the number of real operations also increases. However this increase depends on how the wideband channels are split, i.e. the total number of $50 \mathrm{kHz}$ and $100 \mathrm{kHz}$ channels. The additional complexity due to recombination of $50 \mathrm{kHz}$ channels is relatively small as can be seen in the case of $50 \mathrm{kHz}$ channels only. In comparison the additional complexity due to recombination of $100 \mathrm{kHz}$ channels is significant. Despite this the parallel GDFT-FB only becomes more efficient than the recombined GDFT-FB for a large number of $100 \mathrm{kHz}$ channels. 


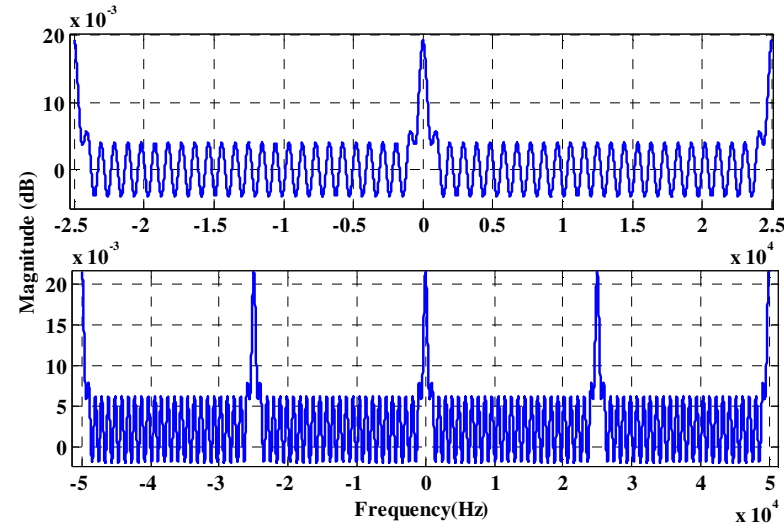

Figure 6 Frequency response pass-band ripple for recombined 50 $\mathrm{kHz}$ and $100 \mathrm{kHz}$ channels

\section{Simulations}

Using the theoretical filter length calculations obtained in the previous section the channelization structures were implemented using MATLAB/Simulink. The frequency responses of a recombined $50 \mathrm{kHz}$ and $100 \mathrm{kHz}$ channels are shown in Figure 6. It can be observed how the pass-band ripple, that had been design to be under $0.1 \mathrm{~dB}$ in the prototype filter, remains within this limit, just showing small peaks in the crossing frequencies between filters. These are produced due to the slightly non-exact $-6 \mathrm{~dB}$ point crossing between adjacent filters. Further prototype filter optimization would lead to constant ripple in the full band.

\section{CONCLUSION}

In this paper two different non-uniform channelization methods have been described and analysed for the specific case of a SDR PMR BS. They were compared based on flexibility to filter channels with different bandwidths and channel spacings, and computational efficiency. The results showed that the computation of the recombined GDFT-FB only exceeds the parallel GDFT-FB when a large number of $100 \mathrm{kHz}$ channels must be recombined. On the other hand, the recombined oversampled filter bank structure is a more flexible scheme that may be applicable to a wider range of radio standards. Finally it has been shown using MATLAB/Simulink that the recombination of channels can be achieved without significant amplitude distortion.

\section{ACKNOWLEDGMENT}

The authors wish to thank Jean-Christophe Schiel and François Montaigne for their assistance and support. Also the authors extend thanks to the sponsors EADS and IRCSET for the $\mathrm{PhD}$ program.

\section{REFERENCES}

R. Mahesh, et al., "Filter Bank Channelizers for Multi-Standard Software Defined Radio Receivers," Journal of Signal Processing Systems, Springer New York, 2008.

F. J. Harris, Multirate Signal Processing for Communication Systems: Prentice Hall PTR, 2004.
P. P. Vaidyanathan, Multirate Systems and Filter Banks: Prentice Hall PTR, 1993.

C. Zhao, et al., "Reconfigurable Architectures for Low Complexity Software Radio Channelizers using Hybrid Filter Banks," in Communication systems, 2006. ICCS 2006. 10th IEEE Singapore International Conference on, 2006, pp. 1-5.

W. A. Abu-Al-Saud and G. L. Stuber, "Efficient wideband channelizer for software radio systems using modulated PR filterbanks," Signal Processing, IEEE Transactions on, vol. 52, pp. 2807-2820, 2004.

ECC, "Public protection and disaster relief spectrum requirements (ECC report 102)," HelsinkiJanuary 2007.

Terrestrial Trunked Radio (TETRA); Feasibility Study, E. T. S. Institute ETSI TR 102513 V1.1.1, 2006-12.

Planning criteria and coordination of frequencies in the land mobile service in the range 29.7-921 MHz, T/R 25-08, 2008.

R. E. Crochiere and L. R. Rabiner, Multirate Digital Signal Processing: Englewood Cliffs (NJ): Prentice Hall, 1983.

A. Palomo Navarro, et al., "Overlapped polyphase DFT modulated filter banks applied to TETRA/TEDS SDR base station channelization," presented at the Royal Irish Academy Communication and Radio Science Colloquium, 2010.

X. M. Xie, et al., "Design of linear-phase recombination nonuniform filter banks," Signal Processing, IEEE Transactions on, vol. 54, pp. 2809-2814, 2006.

F. J. M. G. Harris, R., "A receiver structure that performs simultaneous spectral analysis and time series channelization," in SDR 09 Technical Conference and Product Exposition, 2009. H. Johansson and P. Lowenborg, "Flexible frequency-band reallocation networks using variable oversampled complexmodulated filter banks," EURASIP J. Appl. Signal Process., vol. 2007, pp. 143-143, 2007.

S. Radhakrishnan Pillai and G. H. Allen, "Generalized magnitude and power complementary filters," in Acoustics, Speech, and Signal Processing, 1994. ICASSP-94., 1994 IEEE International Conference on, 1994, pp. III/585-III/588 vol.3.

P. Duhamel and M. Vetterli, "Fast fourier transforms: a tutorial review and a state of the art," vol. 19, ed: Elsevier NorthHolland, Inc., 1990, pp. 259-299.

Xilinx, "LogiCORE IP Fast Fourier Transform v7.1 Product Specification," ed, 2010.

T. Saramäki, "Finite impulse response filter design," in Handbook for Digital Signal Processing, S. K. Mitra and J. F. Kaiser, Eds., ed New York , NY, USA: John Wiley \& Sons, 1993, pp. 155-227.

Q.-G. Liu, et al., "Simple design of oversampled uniform DFT filter banks with applications to subband acoustic echo cancellation," Signal Processing, vol. 80, pp. 831-847, 2000.

A. Viholainen, "Modulated Filter Bank Design for Communication Signal Processing," $\mathrm{PhD}$, Tampere University of Technology, Tampere, 2004. 